\title{
A FLATTENED CLOUD CORE IN NGC 2024
}

P. T. P. HO

Harvard-Smithsonian Center for Astrophysics

Y-L. PENG

Nanjing University, Nanjing, PRC

J. M. TORRELLES

Instituto de Astrofísica de Andalucía, CSIC (Spain), and CfA

J. F. GÓMEZ

Harvard-Smithsonian Center for Astrophysics

L. F. RODRÍGUEZ and J. CANTÓ

Instituto de Astronomía, UNAM (México)

\section{SUMMARY}

With the VLA in the D configuration we have mapped the $(\mathrm{J}, \mathrm{K})=(1,1)$ and $(2,2) \mathrm{NH}_{3}$ lines toward a molecular cloud core in NGC 2024. This region, which contains one of the most highly collimated molecular outflows (Richer et al. 1992), has been studied extensively using a variety of techniques, including dust continuum in the far-infrared (FIR) wavelengths (Mezger et al. 1988, 1992), and molecular lines (see Barnes \& Crutcher 1992 and references therein). We find that the molecular condensations associated with FIR 5, 6, and 7 (Mezger et al. 1988,1992 ) have kinetic temperatures $T_{K} \simeq 40 \mathrm{~K}$. We also find a perturbation of the molecular gas near FIR 6 and FIR 7 in terms of broadening of the ammonia lines. These results suggest that these condensations may not be protostars heated by gravitational energy released during collapse, but that they have an internal heating source. A flattened structure of ammonia emission is found extending parallel to the unipolar $\mathrm{CO}$ outflow structure, but displaced systematically to the east. The location of the high velocity outflow along the surface of the $\mathrm{NH}_{3}$ structure suggests that a wind is sweeping material from the surface of this elongated cloud core. Figure 1 is an overlay of the VLA ammonia emission (dotted area) on top of the $\mathrm{C}^{18} \mathrm{O}$ emission (thick contours) and the $\mathrm{CO}$ outflow (thin contours).

\section{REFERENCES}

Barnes, P. J., \& Crutcher, R. M. 1992, ApJ, 389, 325 
Mezger, P. G., Chini, R., Kreysa, E., Wink, J. E., \& Salter, C. J. 1988, A\&A, 191,44

Mezger, P. G., Sievers, A. W., Haslam, C. G. T., Kreysa, E., Lemke, R., Mauersberger, R., \& Wilson, T. L. 1992, A\&A, 256, 631

Richer, J. S., Hills, R. E., \& Padman, R. 1992, MNRAS, 254, 525

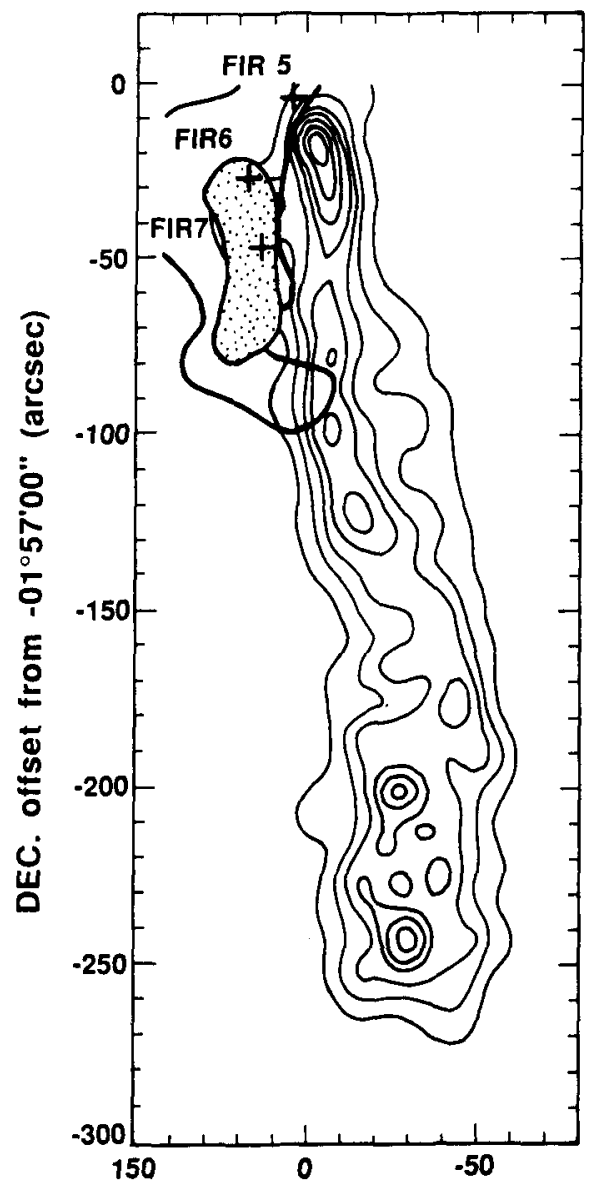

R.A. offset from $05^{h} 39^{m} 12^{s} .6$ (arcsec) 